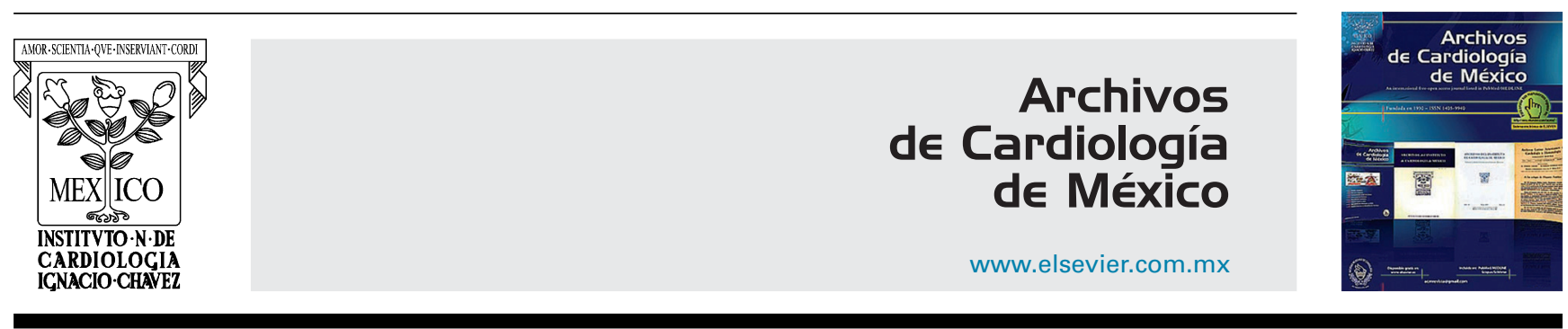

IMAGE IN CARDIOLOGY

\title{
Giant coronary ectasia and athlete's heart mimicking an acute coronary syndrome
}

\section{Ectasia coronaria gigante y corazón de atleta que simulan un síndrome coronario agudo}

\author{
Luis Manautou ${ }^{a}$, Vicente Jimenez ${ }^{a}$, Alejandro Trevino ${ }^{a}$, David Rodriguez ${ }^{a, b}$, \\ Carlos Jerjes-Sanchez ${ }^{a, b, *}$
}

\author{
a Instituto de Cardiología y Medicina Vascular, TEC Salud, San Pedro Garza García, Nuevo León, Mexico \\ b Centro de Investigación Biomédica, Hospital Zambrano Hellion, TEC Salud, Escuela de Medicina, Tecnológico de Monterrey, San \\ Pedro Garza García, Nuevo León, Mexico
}

Received 25 January 2018; accepted 21 March 2018

\begin{abstract}
Coronary artery ectasia is observed in $0.3-5 \%$ of diagnostic angiograms; $>50 \%$ of cases are related to coronary atherosclerosis, $20-30 \%$ are congenital and up to $20 \%$ are related to connective tissue or inflammatory diseases. The impact of strenuous exercise on the left ventricle's structure has been demonstrated. Studies with ECGs and echocardiography discovered a high prevalence of increased cardiac voltage, suggestive of left ventricle enlargement and hypertrophy respectively. ${ }^{1}$ Coronary artery anomalies have been described in $14 \%$ of athletes. ${ }^{2}$ A 37 -year-old man, a retired professional football soccer player with history of syncope upon exertion, presented to the ED with ischemic acute chest pain at rest. On admission, his pulse was 70 beats/min, blood pressure $146 / 85 \mathrm{mmHg}$. Physical examination was normal. ECG revealed ST dynamic changes and negative deep $\mathrm{T}$ waves on anterior-lateral region (Fig. $1 \mathrm{~A}$ and $\mathrm{B}$ ). Laboratory tests revealed normal values of cardiac biomarkers.
\end{abstract}

Immediate diagnostic coronary angiography showed evidence of left main and proximal anterior descending coronary artery ectasia $(12 \mathrm{~mm})$ with intracoronary contrast reflux phenomenon, no obstructive disease or thrombus were observed (Fig. 1C). Transthoracic echocardiography showed mild bilateral atrial dilation, left ventricular hypertrophy without wall motion abnormalities and ejection fraction 56\%. Computed tomography angiography shows coronary ectasia; aorta evaluation with no evidence of dilation, hematoma or dissection (Fig. 1D). Therapy was started with non-fractioned heparin infusion. No abnormalities were found on 24-h Holter monitoring. Clinical and ECG improvement was observed at $48 \mathrm{~h}$. The patient was discharged with oral anticoagulation. This case establishes the relevance of coronary angiography as a diagnostic tool, in those cases in which the diagnosis of ST-elevation myocardial infarction is uncertain.

\footnotetext{
* Corresponding author at: Batallón de San Patricio 112, Real San Agustin, CP 66278, San Pedro Garza García, Nuevo León, Mexico. Tel.: +52 88880000 .

E-mail addresses: jerjes@prodigy.net.mx, carlos.jerjes@udicem.org.mx (C. Jerjes-Sanchez).
} 

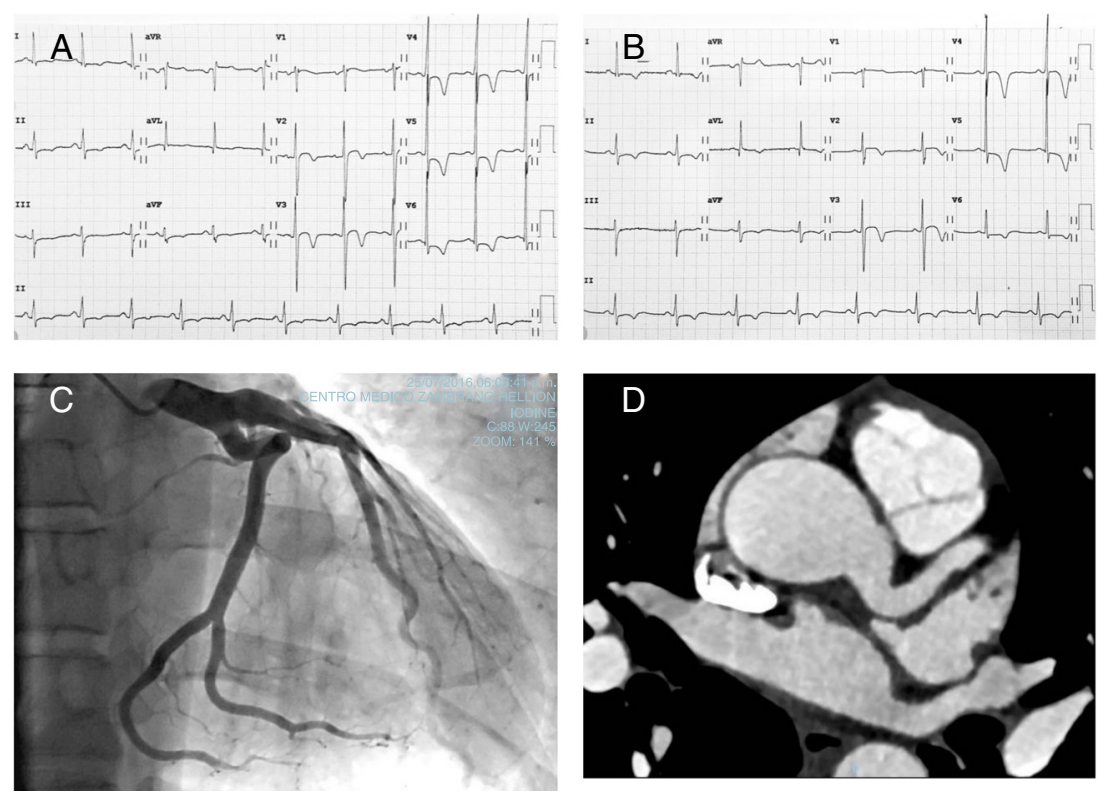

Figure 1 (A) ECG shows isolated increase of QRS amplitude with normal QRS axis and normal atrial activation; V2 to V6 with negative deep T waves and V3 to V6 with ST depression. (B) ECG $48 \mathrm{~h}$ after shows improvement of ST depression. (C and D) Coronary angiogram and computed tomography angiography showed left main $(12 \mathrm{~mm})$ and proximal left anterior descending coronary ectasia.

\section{References}

1. Corrado D, Pelliccia A, Heidbuchel H, et al. Recommendations for interpretation of 12-lead electrocardiogram in the athlete. Eur Heart J. 2010;31:243-59.

2. Maron BJ, Pelliccia A. The heart of trained athletes. Circulation. 2016;114:1633-44. 